\title{
A Web-based Meeting Scheduling Solver with Privacy Guarantees, without Trusted Servers
}

\author{
Marius-Călin Silaghi and Vaibhav Rajeshirke, Richard Wallace \\ Florida Institute of Technology University College Cork
}

Some problems having privacy requirements can be modeled with distributed (weighted) constraint satisfaction frameworks [3]. Previous approaches to such problems used trusted servers or some kind of argumentation, inherently leaking data about the secret constraints [5]. We developed techniques ${ }^{\text {patent pending }}$ and a system for solving these problems where an agent does not divulge absolutely any secret information to any attacker controlling less than half of the participants. Agents and servers only learn a randomly picked solution.

We identify the following privacy attacks on distributed CSP techniques: 1. S-attacks against secure multiparty computation-backtracking hybrids. [1]

2. Shadow CSPs against argumentation based solvers. [5]

3. Attacks against search with known orders on variables and domains. [2]

4. Statistical attacks against DisCSP solvers that shuffle domains. [4]

To achieve resistance to these attacks we invented two fundamental cryptographic techniques: a) S-mixnets for shuffling shared secrets; b) Two alternative functions for solving WCSPs using solely ' $+/^{*}$ ' operations (with no comparison).

We also developed three secure multiparty protocols that combine S-mixnets with the aforementioned functions, obtaining MPC-DisWCSP1 and MPCDisWCSP2 (resistant to attacks 1-3), and MPC-DisWCSP3 (resistant to attacks 1-4). MPC-DisWCSP1 can be parametrized between polynomial space and linear logic time, but is slower. Only MPC-DisWCSP3 can exploit public constraints.

Results: An applet-based secure meeting scheduling system is deployed at www.cs.fit.edu/ ${ }^{\sim}$ msilaghi/secure. The methods also apply for incentive auctions and stable matchings problems [3]. Our solution to the $4^{\text {th }}$ attack can be used to improve the privacy offered by other computation techniques based on DisCSPs (e.g with trusted servers, choosing a solution randomly among all solutions).

\section{References}

1. M. Silaghi. Asynchronously Solving Problems with Privacy Requirements. PhD thesis, EPFL, 2002. www.cs.fit.edu/ ${ }^{\sim}$ msilaghi/teza.

2. M. Silaghi. Solving a DisCSP with cryptographic multi-party computations, without revealing constraints and without involving trusted servers. In IJCAI DCR Workshop, 2003.

3. M. Silaghi. Incentive auctions and stable marriages problems solved with $\lfloor n / 2\rfloor$ privacy of human preferences. Technical Report CS-2004-11, FIT, 2004.

4. M. Silaghi and V. Rajeshirke. The effect of reordering policies on privacy. In AAMAS (to appear), 2004.

5. R. Wallace. Reasoning with possibilities in multiagent graph coloring. In IJCAI DCR Workshop, 2003. 